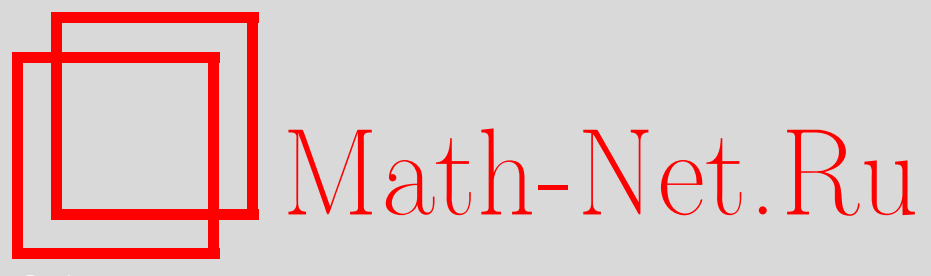

А. Ю. Коняев, Классификация алгебр Ли с орбитами коприсоединенного представления общего положения размерности 2, Матем. сб., 2014, том 205, номер 1, 47-66

DOI: https://doi.org/10.4213/sm8197

Использование Общероссийского математического портала Math-Net.Ru подразумевает, что вы прочитали и согласны с пользовательским соглашением http://www . mathnet.ru/rus/agreement

Параметры загрузки:

IP: 18.209 .158 .208

26 апреля 2023 г., 08:04:19

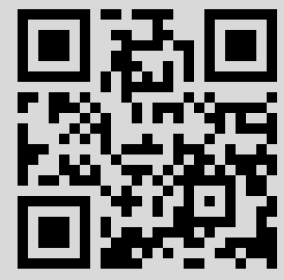




\section{А. Ю. Коняев \\ Классификация алгебр Ли с орбитами коприсоединенного представления общего положения размерности 2}

В работе представлен полный список алгебр Ли над $\mathbb{R}$, орбиты коприсоединенного представления которых в общем положении имеют размерность 2.

Библиография: 20 названий.

Ключевые слова: скобка Пуассона, алгебра Ли, коприсоединенное представление, интегрируемые системы.

DOI: $10.4213 / \mathrm{sm} 8197$

\section{§1. Введение}

Простейшим пуассоновым многообразием является аффинное пространство с постоянной пуассоновой структурой (т.е. постоянным тензором Пуассона) на нем. Симплектические слои такого многообразия суть аффинные плоскости, размерность которых равна рангу тензора Пуассона. С точки зрения геометрии такие скобки малоинтересны, поэтому простейшим нетривиальным пуассоновым многообразием разумно считать аффинное пространство с линейной пуассоновой структурой. Хорошо известно, что в этом случае само аффинное пространство можно отождествить с пространством, двойственным к некоторой алгебре Ли g. Будем обозначать это пространство через $\mathfrak{g}^{*}$. Симплектические слои в этом случае оказываются орбитами коприсоединенного представления группы Ли $G$ с касательной алгеброй Ли $\mathfrak{g}$.

$\mathrm{C}$ точки зрения интегрируемых систем в свою очередь простейшим случаем пуассонова многообразия следует считать многообразие с двумерными симплектическими слоями. Действительно, функции Казимира скобки Пуассона считаются тривиальными интегралами, а гамильтонова система на таком многообразии не требует никаких нетривиальных интегралов.

Эти два замечания приводят нас к формулировке следующей задачи: классифицировать все линейные скобки Пуассона на аффинных пространствах в вещественном случае с симплектическими слоями общего положения размерности 2, или, в алгебраических терминах, описать вещественные алгебры Ли

Работа выполнена в рамках Программы поддержки ведущих научных школ РФ (грант № НШ-1410.2012.1), ФЦП "Научные и научно-педагогические кадры инновационной России" (контракты № 14.740.11.0794 и № 14.740.11.0876), а также гранта Правительства РФ для господдержки научных исследований, проводимых под руководством ведущих ученых (договор № 11.G34.31.0054). 
с орбитами коприсоединенного представления общего положения размерности 2. В таком виде (и с такой же мотивационной частью) эта задача была сформулирована в работе А. Ю. Коняева, А. М. Изосимова, А. В. Болсинова и А. А. Ошемкова [1] под номером 6, а).

Надо сказать, что эта задача имеет не только теоретический интерес. Частным случаем изучаемых алгебр Ли является полупрямая сумма двух коммутативных алгебр Ли $\mathbb{R}$ и $\mathbb{R}^{n}$. В работах И. А. Тайманова и А. В. Болсинова [2], [3] для таких алгебр Ли были построены интегрируемые левоинвариантные геодезические потоки на соответствующих этим алгебрам группах $G$. Такого рода потоки также изучались в работах А. Т. Фоменко, А. В. Браилова, В. В. Трофимова (см. [4]-[10]). Примечательно, что в некоторых случаях возникающие потоки удалось опустить на однородные пространства $G / \Gamma$. Еще одним примечательным фактом, связанным с алгебрами Ли такого рода, является следующий. Тензор Пуассона полупрямой суммы $\mathbb{R}$ и $\mathbb{R}^{n}$ разложим (разложимым скобкам Пуассона посвящен $\S 2$ ), т.е. представляется в виде $v \wedge w$, где векторное поле $v$ постоянно, а $w$ линейно. Ранг тензора Пуассона в точке общего положения равен 2 и падает до нуля только там, где эти два векторных поля зависимы. Из результатов $\S 3$ следует, что это происходит только в точках, где $w=0$. В случае, когда оператор, задающий векторное поле $w$, невырожденный, такие точки образуют одномерную прямую. Для данной алгебры Ли оно является множеством сингулярных элементов и имеет коразмерность $n$, где $n+1$ - размерность всей алгебры Ли. Впервые этот пример был описан в работе Д. И. Панюшева и О. С. Якимовой [11] и принадлежит Э. Винбергу. Коразмерность множества сингулярных элементов играет важную роль при изучении полных коммутативных наборов на алгебрах Ли (см. [12], [11]).

В работе С.А. Шашкова [13] рассматривалась задача классификации геометрически коммутативных неразложимых однородных пространств с одномерным стабилизатором для комплексных алгебраических групп. Классификация проводилась исключительно алгебраическими методами в дополнительном предположении, что соответствующая стабилизатору одномерная подалгебра не является идеалом. Как оказалось, эта задача сводится к поиску пар “алгебра Ли $\mathfrak{g}$ - одномерная подалгебра $\mathfrak{h}$ ” со следующим свойством: для элемента общего положения из Ann h размерность его орбиты коприсоединенного (относительно действия группы Ли $G$ алгебры Ли g) равна 2. Легко понять, что для алгебры $\mathfrak{h}$ общего положения Ann $\mathfrak{h}$ содержит регулярные элементы $\mathfrak{g}^{*}$, поэтому комплексные алгебры Ли со свойством, что орбиты коприсоединенного действия общего положения имеют размерность 2, являются, вообще говоря, частным случаем поставленной задачи. Оказалось, однако, что список алгебр Ли $\mathfrak{g}$, полученный С. А. Шашковым, в точности совпадает с комплексификацией списка, полученного в настоящей работе, т.е. имеются одна бесконечномерная серия $\mathbb{C}+{ }_{\rho} \mathbb{C}^{n}$ и четыре исключительные алгебры Ли: $\operatorname{sl}(2), A_{4,8}, A_{5,3}, A_{6,3}$ (см. также [14]).

Работа организована следующим образом.

В §2 вводится понятие разложимой скобки Пуассона и описываются некоторые свойства таких скобок, многие из которых, равно как и само понятие, 
представляют самостоятельный интерес (например, позволяют описать содержательный класс полиномиальных скобок Пуассона на аффинном пространстве). В $\S 3$ рассматривается класс скобок Пуассона, возникающих на пространстве, двойственном к алгебре Ли, которая представляется в виде полупрямой суммы одномерной коммутативной алгебры Ли и линейного пространства произвольной размерности. Алгебры Ли такого вида изучались в [2], [3], [15]. Также эти алгебры Ли можно рассматривать как частный случай понятия расширения алгебры, описанного, например, в [10]. Некоторые результаты этого параграфа не являются новыми, однако для полноты изложения приводятся с доказательствами. В $\S 4$ представлен основной технический инструмент, используемый при классификации алгебр Ли, который оказывается интересной задачей о свойстве семейств линейных векторных полей на аффинном пространстве. В $\S 5$ описаны центральные расширения алгебр Ли размерности до 3 включительно, этот результат также нужен для классификации. Наконец, в 66 приводится полная классификация алгебр Ли, орбиты коприсоединенного представления общего положения которых имеют размерность 2.

\section{§ 2. Разложимые скобки Пуассона}

Рассмотрим пуассоново многообразие $M^{n}$, снабженное скобкой Пуассона, ранг которой почти всюду равен 2. Будем называть скобку Пуассона разложимой, если на многообразии $M^{n}$ найдется пара таких векторных полей $v$ и $w$, что тензор этой скобки представляется в виде $\mathscr{A}=v \wedge w$. Такого рода скобки возникают, например, в приложениях к механике (см. [16]). Следующее утверждение дает необходимое и достаточное условие того, что пара векторных полей задает тензор Пуассона.

ПреДЛОЖЕНИЕ 1. Пусть на многообразии $M^{n}$ задана пара векторных полей $v, w$. Бивекторное поле $\mathscr{A}=v \wedge w$ является тензором Пуассона тогда и только тогда, когда распределение, задаваемое векторными полями, интегрируемо.

ДокАЗАТЕльство. Бивекторное поле задает скобку Пуассона тогда и только тогда, когда его скобка Схоутена (см. [17]) с собой равна нулю: $[[\mathscr{A}, \mathscr{A}]]=0$. Подставляя в это выражение $\mathscr{A}=v \wedge w$ и применяя свойства, связывающие скобку Схоутена с внешним умножением векторных полей, получаем

$$
[[\mathscr{A}, \mathscr{A}]]=2([v, w] \wedge v \wedge w)=0
$$

где $[v, w]$ обозначает коммутатор векторных полей. Таким образом, тот факт, что $\mathscr{A}$ задает тензор Пуассона, эквивалентен тому, что коммутатор полей $v$ и $w$ разлагается по $v$ и $w$. В свою очередь, по теореме Фробениуса, это равносильно тому, что задаваемое векторными полями $v$ и $w$ распределение интегрируемо. 
Характеристическое распределение $\mathscr{A}$ устроено следующим образом: в точках, где $v$ и $w$ линейно независимы, оно совпадает с распределением, порождаемым этими векторными полями, а во всех остальных точках оно имеет размерность 0. Функции Казимира такой скобки Пуассона - это в точности общие первые интегралы векторных полей $v$ и $w$.

Напомним, что векторное поле $v$ называется гамильтоновым относительно скобки Пуассона $\mathscr{A}$, если для произвольной функции $g$ и некоторой фиксированной функции $f$ выполнено тождество $v(g)=\{g, f\}$, где $v(\cdot)$ обозначает операцию дифференцирования вдоль векторного поля. Функция $f$ называется гамильтонианом векторного поля $v$, а само поле обозначается как $\operatorname{sgrad} f$ (читается: косой градиент $f$ ). Тождество Якоби в терминах косых градиентов переписывается как

$$
[\operatorname{sgrad} f, \operatorname{sgrad} g]=\operatorname{sgrad}\{f, g\}
$$

для произвольных $f$ и $g$. В частности, косые градиенты всех функций задают интегрируемое распределение.

ПреДЛОЖенИЕ 2. Пусть на многообразии $M^{n}$ задана скобка Пуассона $\mathscr{A}$ ранга 2. Возъмем пару функиий $f$ и $g$ и соответствующие им гамильтоновы векторные поля sgrad $f u \operatorname{sgrad} g$. Они определяют разложимую скобку $\mathscr{A}^{\prime}=$ $\operatorname{sgrad} f \wedge \operatorname{sgrad} g$. Эта скобка связана с исходной следующим соотношением:

$$
\mathscr{A}^{\prime}=-\{f, g\} \mathscr{A} \text {. }
$$

ДокАЗАтЕЛЬСтво. Пусть в некоторой точке $x$ поле $\operatorname{sgrad} f$ отлично от нуля (если таких точек нет, то теорема доказана, так как $\{f, g\}=\operatorname{sgrad} f(g)=0)$. По теореме о выпрямлении векторного поля в неособой точке в окрестности $U(x)$ найдется функция $h$ такая, что $\{f, h\}=1$. Легко видеть, что $\operatorname{sgrad} h \neq 0$ в $x$ и дифференциалы $f$ и $h$ независимы в этой точке.

Дополним $f$ и $h$ до локальной системы координат $n-2$ локальными функциями Казимира. Получаем систему координат, в которой $\mathscr{A}=\frac{\partial}{\partial f} \wedge \frac{\partial}{\partial h}$.

В этой системе координат

$$
\operatorname{sgrad} g=\frac{\partial}{\partial h} \frac{\partial}{\partial f}-\frac{\partial g}{\partial f} \frac{\partial}{\partial h} .
$$

В свою очередь

$$
\operatorname{sgrad} g \wedge \operatorname{sgrad} f=-\operatorname{sgrad} g \wedge \frac{\partial}{\partial h}=-\frac{\partial g}{\partial f} \mathscr{A}=-\{f, g\} \mathscr{A} .
$$

Предложение доказано.

Отметим, что в случае, когда ранг скобки почти всюду равен 2, в окрестности точки общего положения по теореме Дарбу тензор Пуассона приводится к постоянному виду $\mathscr{A}=\frac{\partial}{\partial x} \wedge \frac{\partial}{\partial y}$ (где $x, y-$ первые две координатные функции системы координат в окрестности точки общего положения), т.е. всякая скобка ранга 2 в окрестности точки общего положения, рассматриваемой как пуассоново многообразие, разложима. 
ЗАмечание 1. Не всякая скобка Пуассона ранга 2 является разложимой. Рассмотрим структуру Пуассона-Ли на пространстве, двойственном к алгебре Ли so(3). Характеристическое распределение этой структуры - касательные плоскости к семейству концентрических сфер с началом в $(0,0,0)$. Из теоремы о непричесывании ежа следует, что на сфере не существует пары векторных полей, всюду порождающих все касательное пространство. Из этого вытекает, что на всем пространстве не существует пары векторных полей, порождающих характеристическое распределение во всех точках, где оно имеет размерность 2. Таким образом, скобка на so(3)* не является разложимой.

Следующее замечание позволяет строить большое количество примеров разложимых скобок.

ЗАмечание 2. Рассмотрим многообразие $M^{n} \times \mathbb{R}$ (или $M^{n} \times S^{1}$, если требуется сохранить компактность). Пусть $v$ и $w$ - векторные поля на $M^{n}$ и $\mathbb{R}$ $\left(\right.$ или $\left.S^{1}\right)$. Продолжим их на произведение таким образом, чтобы в локальной системе координат, согласованной с этим произведением, поле $v$ не зависело от координат на $\mathbb{R}$ (или на $S^{1}$ ), а $w$ - от координат на $M^{n}$. Легко видеть, что $[v, w]=0$, поэтому $\mathscr{A}=v \wedge w$ задает скобку Пуассона.

Следующее предложение, вообще говоря, не будет использоваться дальше, однако представляет самостоятельный интерес.

ПрЕДЛОЖЕНИЕ 3. Пусть на гладком (аналитическом, алгебраическом) многообразии $M^{n}$ задана гладкая (аналитическая, алгебрачческая) скобка Пуассона, ранг которой почти всюду равен 2. Обозначим через $\mathscr{A}$ тензор Пуассона данной скобки. Для любой гладкой (аналитической, алгебраической) функиии $f$ на многообразии $M^{n}$ верно, что $f \mathscr{A}$ также является скобкой Пуассона, причем согласованной с исходной.

ДоказАтельство. Рассмотрим точку общего положения. По теореме Дарбу в окрестности этой точки существует такая система координат, что $\mathscr{A}=$ $\frac{\partial}{\partial x} \wedge \frac{\partial}{\partial y}$, где $x, y-$ первые две координатные функции системы. Рассмотрим эту окрестность как пуассоново многообразие, на котором задана пара векторных полей $f \frac{\partial}{\partial x}$ и $\frac{\partial}{\partial y}$ (через $f$ в данном случае мы обозначаем ограничение функции $f$ со всего многообразия на окрестность). Коммутатор этих полей имеет вид

$$
\left[f \frac{\partial}{\partial x}, \frac{\partial}{\partial y}\right]=-\frac{\partial f}{\partial y} \frac{\partial}{\partial x} .
$$

Из этого следует, что задаваемое векторными полями распределение интегрируемо. По предложению 1 получаем, что бивекторное поле $f \frac{\partial}{\partial x} \wedge \frac{\partial}{\partial y}=f \mathscr{A}$ задает скобку Пуассона.

Напомним, что пара скобок называется согласованной, если любая их линейная комбинация с постоянными коэффициентами снова является скобкой Пуассона. В нашем случае $\lambda \mathscr{A}+\mu f \mathscr{A}=(\lambda+\mu f) \mathscr{A}$. В силу произвольности выбора $f$ получаем, что полученная скобка - скобка Пуассона, а значит, исходные скобки согласованы. 
Тот факт, что $f \mathscr{A}$ является скобкой Пуассона, эквивалентен равенству нулю скобки Схоутена этого тензора с собой: $[[f \mathscr{A}, f \mathscr{A}]]=0$. В силу приведенных рассуждений это равенство выполнено почти всюду. Продолжая его по непрерывности, получаем, что оно выполнено уже везде и $f \mathscr{A}$ задает скобку Пуассона.

ЗАмЕчАниЕ 3. В случае, когда $v$ и $w$ - пара полиномиальных векторных полей на линейном пространстве (т.е. пара векторных полей, координаты которых - полиномиальные функции), задающих интегрируемое распределение, тензор $\mathscr{A}=v \wedge w$ представляет собой полиномиальную скобку Пуассона.

\section{§ 3. Полупрямые суммы $\mathbb{R}$ и $\mathbb{R}^{n}$}

Основным объектом изучения данного параграфа являются алгебры Ли вида $\mathfrak{g}=\mathbb{R}+{ }_{\rho} \mathbb{R}^{n}$. Зафиксируем в $\mathfrak{g}$ базис $e_{1}, \ldots, e_{n+1}$ такой, что $e_{2}, \ldots, e_{n+1}$ образуют коммутативный идеал. Тензор Пуассона на $\mathfrak{g}^{*}=\mathbb{R}^{*}+\mathbb{R}^{n *}$ такой алгебры разложим, он представляется в виде $\mathscr{A}=v \wedge w$, где $v(x)=\frac{\partial}{\partial e_{1}}$, а $w(x)=\operatorname{ad}_{e_{1}}^{*} x$, где $x \in \mathfrak{g}^{*}$.

Обозначим через $\mathfrak{b}_{2}$ некоммутативную двумерную алгебру Ли, а через $\mathfrak{h}_{3}-$ трехмерную алгебру Гейзенберга, т.е. алгебру со следующими коммутационными соотношениями в подходящем базисе $e_{1}, e_{2}, e_{3}$ :

$$
\left[e_{1}, e_{2}\right]=\left[e_{3}, e_{2}\right]=0, \quad\left[e_{1}, e_{3}\right]=e_{2} .
$$

Следующая теорема является основным результатом данного параграфа и описывает важное характеристическое свойство алгебр Ли вида $\mathfrak{g}=\mathbb{R}+{ }_{\rho} \mathbb{R}^{n}$.

ТеОрема 1. Алгебра Ли $\mathfrak{g}$ размерности $n+1$ представляется в виде $\mathbb{R}+{ }_{\rho} \mathbb{R}^{n}$ тогда и только тогда, когда в ней существует коммутативная подалгебра размерности $n$.

ДокАЗАтЕльство. В одну сторону утверждение теоремы очевидно: в качестве коммутативной подалгебры размерности $n$ в $\mathbb{R}+{ }_{\rho} \mathbb{R}^{n}$ можно взять идеал $\mathbb{R}^{n}$.

Покажем, что утверждение выполнено в другую сторону. Пусть алгебра Ли некоммутативна (иначе утверждение теоремы очевидно). Выберем базис $e_{1}, \ldots, e_{n}, f$ такой, что $e_{i}$ образуют базис в $n$-мерной коммутативной подалгебpe, существующей по условию теоремы. Считаем, что в двойственном пространстве $\mathfrak{g}$ фиксирован базис, двойственный к данному, который мы обозначим через $\frac{\partial}{\partial e_{i}}, \frac{\partial}{\partial f}$.

Лемма 1. Пусть в алгебре Ли $\mathfrak{g}$ размерности $n+1$, отличной от $\mathfrak{b}_{2} \oplus \mathbb{R}^{n-1}$, имеется коммутативная подалгебра размерности $n$. Тогда эта подалгебра идеал.

ДокАЗАтЕЛЬство. Обозначим через $v$ векторное поле $\operatorname{sgrad} f$. По построению это векторное поле линейно и отлично от нуля, так как алгебра Ли $\mathfrak{g}$ не коммутативна с векторным полем. В этих координатах $\mathscr{A}=\frac{\partial}{\partial f} \wedge v$. 
Рассмотрим коммутатор (в смысле векторных полей) $w=\left[\frac{\partial}{\partial f}, v\right]$. Легко видеть, что $w(f)=\frac{\partial}{\partial f}(\{f, f\})-v(1)=0$ и, следовательно, $w$ линейно независимо с $\frac{\partial}{\partial f}$. Из теоремы Фробениуса и предложения 1 вытекает, что $w \wedge v=0$.

Если $w=0$, то компоненты векторного поля $v$ не зависят от $f$ и, следовательно, $\left[f, e_{i}\right]$ разлагается по $e_{i}$. Таким образом, подалгебра, натянутая на $e_{i},-$ идеал.

Пусть $w \neq 0$. Покажем, что в этом случае $\mathfrak{g}$ изоморфно $\mathfrak{b}_{2} \oplus \mathbb{R}^{n-1}$. Обозначим

$$
w=w_{1} \frac{\partial}{\partial e_{1}}+\cdots+w_{n} \frac{\partial}{\partial e_{n}} .
$$

Без ограничения общности считаем, что $w_{i} \neq 0$ для $i=1, \ldots, k$ и $w_{i}=0$ для $i>k$. Аналогичным образом, обозначим

$$
v=v_{1} \frac{\partial}{\partial e_{1}}+\cdots+v_{n} \frac{\partial}{\partial e_{n}} .
$$

Напомним, что $w_{i}$ - константы, а $v_{i}$ - линейные функции.

Рассмотрим два случая.

Случай $k=1$. Условие

$$
0=v \wedge w=w_{1} v_{i} \frac{\partial}{\partial e_{1}} \wedge \frac{\partial}{\partial e_{i}}
$$

дает $v_{i}=0$ для $i \geqslant 2$. Выполним замену базиса

$$
\widetilde{e}_{2}=\frac{1}{w_{2}} e_{2}, \quad \widetilde{e}_{1}=e_{1}+\frac{1}{w_{2}} v_{2} .
$$

Коммутационные соотношения в новом базисе имеют вид $\left\{\widetilde{e}_{1}, \widetilde{e}_{2}\right\}=\widetilde{e}_{1}$, а скобки Пуассона остальных базисных элементов равны нулю. Таким образом, $\mathfrak{g}$ изоморфна $\mathfrak{b}_{2} \oplus \mathbb{R}^{n-1}$.

Случай $k>1$. Рассмотрим компоненты $v \wedge w$ с номерами $(2, j)$ при $2 \leqslant i<$ $j \leqslant k$. Они имеют вид $w_{i} v_{2}-w_{2} v_{i}$ и по условию равны нулю. Выполним замену координат

$$
\widetilde{e}_{2}=\frac{1}{w_{2}} e_{2}, \quad \widetilde{e}_{j}=e_{j}-\frac{w_{j}}{w_{2}} e_{2}, \quad 3 \leqslant j \leqslant k, \quad \widetilde{e}_{1}=e_{1}+\frac{1}{w_{2}} v_{1} .
$$

В новой системе координат $\left\{\widetilde{e}_{1}, \widetilde{e}_{2}\right\}=\widetilde{e}_{1}$, а скобки Пуассона остальных элементов базиса равны нулю. В этом случае $\mathfrak{g}$ также изоморфна $\mathfrak{b}_{2} \oplus \mathbb{R}^{n-1}$.

Так как $\mathfrak{g}$ не изоморфно $\mathfrak{b}_{2} \oplus \mathbb{R}^{n-1}$ по условию леммы, то $w=0$. Таким образом, лемма доказана.

Теперь рассмотрим алгебру Ли $\mathfrak{g}$ размерности $n+1$. Пусть она содержит коммутативную подалгебру размерности $n$. Если алгебра $\mathfrak{g}$ изоморфна $\mathfrak{b}_{2} \oplus$ $\mathbb{R}^{n-1}$, то она представляется в виде полупрямой суммы одномерной алгебры Ли и коммутативного идеала. Если $\mathfrak{g}$ не изоморфны $\mathfrak{b}_{2} \oplus \mathbb{R}^{n-1}$, то по доказанной 
лемме эта коммутативная подалгебра - идеал. Таким образом, $\mathfrak{g}$ - полупрямая сумма одномерной алгебры Ли и коммутативного идеала.

Теорема 1 доказана.

Будем говорить, что два линейных оператора $R_{1}$ и $R_{2}$ эквивалентны, если найдется такая ненулевая константа $\mu$, что нормальные жордановы формы операторов $R_{1}$ и $\mu R_{2}$ совпадают. Будем говорить, что два представления $\rho_{1}$ и $\rho_{2}$ одномерной алгебры Ли $\mathbb{R}$ в $\mathrm{gl}(V)$ эквивалентны, если для произвольного $e_{1} \in \mathbb{R}$ операторы $\rho_{1}\left(e_{1}\right)$ и $\rho_{2}\left(e_{1}\right)$ эквивалентны. Следующая теорема приводится в работе [15].

ТЕОРема 2. Пусть задана пара алгебр Ли вида $\mathfrak{g}_{1}=\mathbb{R}+_{\rho_{1}} \mathbb{R}^{n}$ u $\mathfrak{g}_{2}=$ $\mathbb{R}+{ }_{\rho_{2}} \mathbb{R}^{n}$. Они изоморбнь тогда и только тогда, когда представления $\rho_{1}$ и $\rho_{2}$ эквивалентны.

\section{§4. Одна задача о линейных векторных полях}

Рассмотрим следующую задачу, касающуюся линейных векторных полей, которая представляет самостоятельный интерес. Пусть на $\mathbb{R}^{n}$ задано семейство из $k+1$ линейных векторных полей. Линейные операторы, соответствующие этим полям, обозначим через $P_{1}, \ldots, P_{k+1}$. Предположим, что семейство векторных полей обладает следующим свойством: почти всюду на $\mathbb{R}^{n}$ размерность касательного пространства, порождаемого этими полями, равна $k$. Нужно ответить на вопрос: какие условия данное свойство накладывает на операторы $P_{i}$ ? Например, описанное условие заведомо выполнено, если операторы $P_{i}$ порождают в линейном пространстве операторов подпространство размерности $k$, т.е. один из операторов выражается через остальные.

Следующая теорема дает ответ на вопрос для случая $k=1$ (именно этот результат потребуется для классификации алгебр Ли с орбитами коприсоединенного представления общего положения размерности 2).

Теорема 3. Пусть на пространстве $\mathbb{R}^{n}$ заданы два линейных векторных поля $v$ u $w$, почти всюду порождающих пространство размерности 1. Обозначим линейные операторы, задающие эти поля, как $P_{1}$ и $P_{2}$. Тогда выполняется, как минимум, одно из двух следующих условий:

1) векторные поля удовлетворяют соотношению $w=\mu v$, где $\mu \in \mathbb{R}$; как следствие, линейно зависимы операторы $P_{1}$ и $P_{2}$, причем $P_{2}=\mu P_{1}$;

2) векторные поля удовлетворяют соотношению $v=a l(x), w=a p(x)$, где а - некоторый постоянный вектор, а $l(x), p(x)$ - линейные функциональ на $\mathbb{R}^{n} ;$ как следствие, образы операторов $P_{1}$ и $P_{2}$ лежат в одном одномерном пространстве, натянутом на вектор а.

ДокАзАтельство. Из того факта, что размерность пространства, порождаемого $v$ и $w$, почти всюду равна 1, следует, что, во-первых, оба поля не могут быть одновременно нулевыми. Без ограничения общности считаем, что $v \neq 0$. Во-вторых, $v \wedge w=0$. Обозначим компоненты векторных полей через $l_{1}(x), \ldots, l_{n}(x)$ и $p_{1}(x), \ldots, p_{n}(x)$ соответственно. В дальнейшем ради экономии 
места зависимость от $x$ указывать не будем. Считаем, что векторы выписаны один под другим в матрицу $2 \times n$, где $v$ сверху.

Если $w=0$, то утверждение доказано, причем $\mu=0$. Пусть не все $p_{i}$ равны нулю. Без ограничения общности считаем, что $p_{1}, \ldots, p_{k}$ для некоторого $k \geqslant 1$ не равны нулю, а $p_{k+1}, \ldots, p_{n}$ тождественно нулевые. Для $j \geqslant k+1$ рассмотрим минор

$$
\left|\begin{array}{rr}
l_{1} & l_{j} \\
p_{1} & 0
\end{array}\right|
$$

Так как $v \wedge w=0$, то этот минор равен нулю и, следовательно, $l_{j}=0$. Таким образом, $l_{k+1}, \ldots, l_{n}$ также тождественно равны нулю. Теперь если $k=1$, то, положив $a=(1,0, \ldots, 0), l(x)=l_{1}(x)$ и $p(x)=p_{1}(x)$, получаем, что выполнен п. 2) теоремы.

Пусть $k \geqslant 2$, и рассмотрим $1<j \leqslant k$. Из равенства нулю аналогичного минора в формуле (4.1) получаем тождество

$$
l_{1} p_{j}=l_{j} p_{1}
$$

Их этого тождества получаем, что $l_{j} p_{1}$ делится на $l_{1}$. В силу неприводимости линейных многочленов над $\mathbb{R}$ получаем, что возможны два варианта.

1. Пусть $p_{1}$ делится на $l_{1}$. Так как оба многочлена линейны, то $p_{1}=\mu l_{1}$. Подставляя это в формулу (4.2) и учитывая, что в формуле фигурирует тождество, получаем $p_{j}=\mu l_{j}$. Таким образом, $w=\mu v$ и выполняется п. 1) теоремы.

2. Пусть $p_{1}$ не делится на $l_{1}$. В этом случае из тождества (4.2) вытекает, что (в силу той же неприводимости линейных многочленов) для любого $j \geqslant 2$ многочлен $l_{j}$ делится на $l_{1}$. Обозначим $l_{j}=a_{j} l_{1}$, где $a_{j}$ - некоторая константа. Из тождества (4.2) получаем, что $p_{j}=a_{j} p_{1}$. Обозначив $a=a_{1}, \ldots, a_{k}, 0, \ldots, 0$, $l_{1}(x)=l(x)$ и $p_{1}(x)=p(x)$, получаем $v=a l(x), w=a p(x)$.

Таким образом, теорема 3 доказана.

\section{§5. Центральные расширения алгебр Ли малой размерности}

В этом параграфе мы рассмотрим центральные расширения алгебр Ли размерностей 2 и 3 . Для этого сначала опишем алгебры Ли этих размерностей. В размерности 2 существует ровно одна некоммутативная алгебра Ли - это $\mathfrak{b}_{2}$. В подходящем базисе $e_{1}, e_{2}$ единственное коммутационное соотношение в этой алгебре имеет вид $\left[e_{1}, e_{2}\right]=e_{2}$. Двумерную коммутативную алгебру Ли мы будем обозначать $\mathfrak{d}_{2}$.

Трехмерные алгебры Ли были классифицированы Л. Бьянки в [18]. Их можно разбить на два семейства с континуальным параметром и шесть отдельных алгебр Ли (табл. 1), т.е. всякая трехмерная алгебра Ли определяется номером класса и значением континуального параметра, если таковой есть. Классификация устроена таким образом, что алгебры Ли, для которых отличается хотя бы один из этих параметров, не изоморфны.

Все результаты этого параграфа относительно просты, однако для полноты изложения приводятся с доказательствами. 
ТАБлицА 1. Трехмерные вещественные алгебры Ли

\begin{tabular}{|c|c|c|}
\hline Обозначение & $\begin{array}{c}\text { Коммутационные соотношения } \\
\text { (ненулевые) }\end{array}$ & Описание \\
\hline$T_{0}$ & - & коммутативная \\
\hline$T_{1}\left(\mathfrak{h}_{3}\right)$ & {$\left[e_{2}, e_{3}\right]=e_{1}$} & нильпотентная \\
\hline$T_{2}$ & $\begin{array}{c}{\left[e_{1}, e_{3}\right]=e_{1}} \\
{\left[e_{2}, e_{3}\right]=e_{1}+e_{2}}\end{array}$ & разрешимая \\
\hline$T_{3, \alpha}$ & $\begin{array}{c}{\left[e_{1}, e_{3}\right]=e_{1}} \\
{\left[e_{2}, e_{3}\right]=\alpha e_{2}} \\
0<|a| \leqslant 1\end{array}$ & разрешимая \\
\hline$T_{4, \alpha}$ & $\begin{array}{c}{\left[e_{1}, e_{3}\right]=\alpha e_{1}-e_{2},} \\
{\left[e_{2}, e_{3}\right]=e_{1}+a e_{2},} \\
a \geqslant 0\end{array}$ & общего типа \\
\hline$T_{5}$ & $\begin{array}{c}{\left[e_{1}, e_{2}\right]=e_{3}} \\
{\left[e_{1}, e_{3}\right]=-e_{2}} \\
{\left[e_{2}, e_{3}\right]=e_{1}}\end{array}$ & $\begin{array}{c}\text { простая, } \\
\text { изоморфная so(3) }\end{array}$ \\
\hline$T_{6}$ & $\begin{array}{c}{\left[e_{1}, e_{2}\right]=2 e_{2},} \\
{\left[e_{1}, e_{3}\right]=-2 e_{3},} \\
{\left[e_{2}, e_{3}\right]=e_{1}}\end{array}$ & $\begin{array}{c}\text { простая, } \\
\text { изоморфная } \operatorname{sl}(2)\end{array}$ \\
\hline$T_{7}$ & {$\left[e_{1}, e_{2}\right]=e_{2}$} & изоморфная $\mathfrak{b}_{2} \oplus \mathbb{R}$ \\
\hline
\end{tabular}

Дадим сначала несколько определений. Центральным расширением $\mathfrak{f}$ алгебры Ли $\mathfrak{g}$ называется алгебра Ли с центром $\mathfrak{a}$, обладающая свойством, что $\mathfrak{f} / \mathfrak{a}=\mathfrak{g}$.

В дальнейшем нам потребуется изучать алгебры Ли с точностью до некоторого отношения эквивалентности. Будем говорить, что алгебры Ли $\mathfrak{f}$ и $\mathfrak{f}^{\prime}$ эквивалентны с точностъю до суммы с коммутативной алгеброй Ли (или просто эквивалентны), если одна из этих алгебр Ли представима в виде прямой суммы другой алгебры Ли и центра, т.е. без ограничения общности $\mathfrak{f}^{\prime}=\mathfrak{f} \oplus \mathbb{R}^{k}$. Класс эквивалентности мы будем обозначать представителем минимальной размерности (легко показать, что такой представитель единственный).

Напомним, что центральное расширение $\mathfrak{f}$ алгебры Ли $\mathfrak{g}$ тривиально, если оно имеет вид $\mathfrak{g} \oplus \mathbb{R}^{k}$. В формулировке теоремы для маломерных алгебр Ли используются обозначения из [19], [20].

Теорема 4. В табл. 2 представлены классы эквивалентности всех нетривиальных иентральных расширений трехмерных и двумерных алгебр Ли. Указаны представители каждого класса минимальной размерности.

Докажем сначала следующую лемму. 
ТАБлицА 2. Классы эквивалентности нетривиальных центральных расширений маломерных алгебр Ли

\begin{tabular}{|c|c|}
\hline Обозначение & $\begin{array}{c}\text { Класс } \\
\text { эквивалентности }\end{array}$ \\
\hline $\mathfrak{d}_{2}$ & $\mathfrak{h}_{3}$ \\
\hline$T_{7}$ & $\mathfrak{b}_{2}, A_{4,3}$ \\
\hline$T_{4,0}$ & $A_{4,10}$ \\
\hline$T_{3,-1}$ & $A_{4,8}$ \\
\hline$T_{1}$ & $A_{5,3}, A_{4,1}$ \\
\hline$T_{0}$ & $A_{6,3}, A_{5,1}, T_{1}$ \\
\hline
\end{tabular}

ЛЕмма 2. Пусть заданы алгебра Ли $\mathfrak{g}$ размерности $n$ и ее центральное расширение f размерности $n+p$. Это центральное расширение эквивалентно некоторой алгебре Ли размерности не больше $n(n+1) / 2$.

ДокАзАТЕЛьство. Утверждение леммы имеет смысл, только если $n+p>$ $n(n+1) / 2$, поэтому предположим, что данное неравенство выполнено. Зафиксируем в $\mathfrak{f}$ базис $e_{1}, \ldots, e_{n}, a_{1}, \ldots, a_{p}$ такой, что $e_{i}$ порождают алгебру Ли, изоморфную $\mathfrak{g}$. В свою очередь $a_{i}$ задают базис в коммутативной подалгебре $\mathfrak{a}$, лежащей в центре $\mathfrak{f}$. Ненулевые коммутационные соотношения в центральном расширении имеют вид

$$
\left[e_{i}, e_{j}\right]=c_{i j}^{k} e_{k}+d_{i j}^{l} a_{l}, \quad 1 \leqslant k \leqslant n, \quad 1 \leqslant l \leqslant p .
$$

Обозначим $f_{i j}=d_{i j}^{l} a_{l}$, а $\mathfrak{a}^{\prime}-$ подпространство в $\mathfrak{a}$, порождаемое $f_{i j}$. По построению размерность этого пространства не превосходит $n(n-1) / 2$. Без ограничения общности считаем, что базис в $\mathfrak{a}$ выбран так, что первые $a_{1}, \ldots, a_{q}$ образуют базис в $\mathfrak{a}^{\prime}$. Как следствие, $\mathfrak{f}$ распадается в прямую сумму алгебры Ли $\mathfrak{f}^{\prime}$, натянутой на $e_{1}, \ldots, e_{n}, a_{1}, \ldots, a_{q}$, и коммутативной алгебры Ли, натянутой на $a_{q+1}, \ldots, a_{p}$. Лемма доказана.

ДоКАЗАТЕЛЬСтво тЕОРЕМЫ 4. Рассмотрим произвольное центральное расширение $\mathfrak{f}$ алгебры Ли $\mathfrak{b}_{2}$. По лемме 2 это расширение эквивалентно некоторой алгебре Ли $\mathfrak{f}^{\prime}$ размерности не более 3 , коммутационные соотношения которой в базисе имеют вид

$$
\left[e_{1}, e_{2}\right]=e_{2}+\lambda a_{1} .
$$

Выполним замену координат $e_{1}^{\prime}=e_{1}, e_{2}^{\prime}=e_{2}+\lambda a_{1}, a_{1}^{\prime}=a_{1}$. В штрихованных координатах коммутационные соотношения принимают вид $\left[e_{1}^{\prime}, e_{2}^{\prime}\right]=e_{2}^{\prime}$, т.е. $\mathfrak{f}^{\prime}=\mathfrak{b}_{2} \oplus \mathbb{R}$ и, следовательно, центральное расширение эквивалентно $\mathfrak{b}_{2}$.

Рассмотрим двумерную коммутативную алгебру Ли. По лемме 2 это расширение эквивалентно некоторой алгебре Ли $\mathfrak{f}^{\prime}$ размерности не более 3 . Пусть в исходной алгебре Ли зафиксирован базис $e_{1}, e_{2}$ и $a_{1}-$ базис центра. Тогда структурные константы имеют вид $\left[e_{1}, e_{2}\right]=a_{1}$, т.е. это трехмерная алгебра Ли $T_{1}$ или $\mathfrak{h}_{3}$. 
Рассмотрим трехмерные алгебры из табл. 1.

Расширение $T_{7}$. В общем виде центральное расширение $T_{7}$ записывается в следующем виде:

$$
\left[e_{1}, e_{2}\right]=e_{2}+\lambda a_{2}, \quad\left[e_{1}, e_{3}\right]=\mu a_{3}, \quad\left[e_{2}, e_{3}\right]=\nu a_{1},
$$

где $a_{i}$ порождают $\mathfrak{a}$. Запишем тождество Якоби для такого расширения для векторов $e_{1}, e_{2}, e_{3}$. Оно имеет вид

$$
\nu a_{1}=0
$$

т.е. $\nu=0$. Рассмотрим два случая.

Первый случай: $\mu \neq 0$. Выполним замену

$$
e_{1}^{\prime}=e_{1}, \quad e_{2}^{\prime}=e_{2}+\lambda a_{2}, \quad e_{3}^{\prime}=e_{3}, \quad a_{1}^{\prime}=a_{1}, \quad a_{2}^{\prime}=a_{2}, \quad a_{3}^{\prime}=\mu a_{3} .
$$

В штрихованной системе координат полученная алгебра Ли изоморфна $A_{4,3} \oplus \mathbb{R}^{2}$, где $A_{4,3}$ - из списка работы [19].

Второй случай: $\mu=0$. Алгебра Ли изоморфна $\mathfrak{b}_{2} \oplus \mathbb{R}^{4}$.

Расширения $T_{6}, T_{5}$. Так как у полупростых алгебр Ли есть только тривиальные центральные расширения, то полученные алгебры Ли будут эквивалентны $\operatorname{sl}(2)$ и so(3), т.е. исходным алгебрам.

Расширение $T_{4, \alpha}$. В общем виде центральное расширение $T_{4, \alpha}$ записывается следующим образом:

$$
\left[e_{1}, e_{3}\right]=\alpha e_{1}-e_{2}+\lambda a_{1}, \quad\left[e_{2}, e_{3}\right]=e_{1}+\alpha e_{2}+\mu a_{2}, \quad\left[e_{1}, e_{2}\right]=\nu a_{3}, \quad \alpha \geqslant 0,
$$

где $a_{i}$ порождают $\mathfrak{a}$.

Запишем для этой алгебры Ли тождество Якоби для векторов $e_{1}, e_{2}, e_{3}$ :

$$
\begin{aligned}
& {\left[e_{1},\left[e_{2}, e_{3}\right]\right]+\left[e_{3},\left[e_{1}, e_{2}\right]\right]+\left[e_{2},\left[e_{3}, e_{1}\right]\right]} \\
& \quad=\left[e_{1}, e_{1}+\alpha e_{2}+\mu a_{2}\right]+\left[e_{3}, \nu a_{3}\right]+\left[e_{2},-\alpha e_{1}+e_{2}-\lambda a_{1}\right] \\
& \quad=2 \alpha\left[e_{1}, e_{2}\right]=2 \alpha \nu a_{3} .
\end{aligned}
$$

Возможны два случая.

Первый случай: $\nu=0$. Рассмотрим замену

$$
e_{1}^{\prime}=e_{1}+\frac{\alpha \lambda a_{1}+\mu a_{2}}{1+\alpha^{2}}, \quad e_{2}^{\prime}=e_{2}+\frac{-\lambda a_{1}+\alpha \mu a_{2}}{1+\alpha^{2}}, \quad e_{3}^{\prime}=e_{3} .
$$

Переход к штрихованному базису показывает, что данное центральное расширение эквивалентно самой алгебре Ли $T_{4, \alpha}$.

Второй случай: $\alpha=0, \nu \neq 0$. Замена координат

$$
e_{1}^{\prime}=e_{1}+a_{2}, \quad e_{2}^{\prime}=e_{2}-\lambda a_{1}, \quad e_{3}^{\prime}=e_{3}
$$

показывает, что данное центральное расширение эквивалентно четырехмерной алгебре Ли $A_{4,10}$ из списка работы [19], в частности эти алгебры Ли изоморфны. 
Расширение $T_{3, \alpha}$. В общем виде центральное расширение $T_{3, \alpha}$ записывается следующим образом:

$$
\left[e_{1}, e_{3}\right]=e_{1}+\lambda a_{1}, \quad\left[e_{2}, e_{3}\right]=\alpha e_{2}+\mu a_{2}, \quad\left[e_{1}, e_{2}\right]=\nu a_{3}, \quad 0<|a| \leqslant 1,
$$

где $a_{i}$ порождают a. Выписываем тождество Якоби для тройки $e_{1}, e_{2}, e_{3}$ :

$$
\begin{aligned}
& {\left[e_{1},\left[e_{2}, e_{3}\right]\right]+\left[e_{3},\left[e_{1}, e_{2}\right]\right]+\left[e_{2},\left[e_{3}, e_{1}\right]\right]} \\
& \quad=\left[e_{1}, \alpha e_{2}+\mu a_{2}\right]+\left[e_{3}, \nu a_{3}\right]+\left[e_{2},-e_{1}-\lambda a_{1}\right] \\
& \quad=2 \alpha\left[e_{1}, e_{2}\right]=(\alpha+1) \nu a_{3} .
\end{aligned}
$$

Первый случай: $\nu=0$. Рассмотрим замену

$$
e_{1}^{\prime}=e_{1}+\lambda a_{1}, \quad e_{2}^{\prime}=e_{1}+\frac{1}{\alpha} \mu a_{2}, \quad e_{3}^{\prime}=e_{3} .
$$

Переход к штрихованному базису показывает, что данное центральное расширение эквивалентно самой алгебре Ли $T_{3, \alpha}$.

Второй случай: $\alpha=-1, \nu \neq 0$. Замена координат

$$
e_{1}^{\prime}=e_{1}+\lambda a_{1}, \quad e_{2}^{\prime}=e_{1}-\mu a_{2}, \quad e_{3}^{\prime}=e_{3}
$$

показывает, что данное расширение эквивалентно четырехмерной алгебре Ли $A_{4,8}$ из списка работы [19], в частности эти алгебры Ли изоморфны.

Расширение $T_{2}$. В общем виде центральное расширение $T_{2}$ записывается следующим образом:

$$
\left[e_{1}, e_{3}\right]=e_{1}+\lambda a_{1}, \quad\left[e_{2}, e_{3}\right]=e_{1}+e_{2}+\mu a_{2}, \quad\left[e_{1}, e_{2}\right]=\nu a_{3},
$$

где $a_{i}$ порождают $\mathfrak{a}$. Запишем тождество Якоби для тройки $e_{1}, e_{2}, e_{3}$ :

$$
\begin{aligned}
& {\left[e_{1},\left[e_{2}, e_{3}\right]\right]+\left[e_{3},\left[e_{1}, e_{2}\right]\right]+\left[e_{2},\left[e_{3}, e_{1}\right]\right]} \\
& \quad=\left[e_{1}, e_{1}+e_{2}+\mu a_{2}\right]+\left[e_{3}, \nu a_{3}\right]+\left[e_{2},-e_{1}-\lambda a_{1}\right] \\
& \quad=2\left[e_{1}, e_{2}\right]=2 \nu a_{3} .
\end{aligned}
$$

Таким образом, $\nu=0$. Выполнив замену

$$
e_{1}^{\prime}=e_{1}+\lambda a_{1}, \quad e_{2}^{\prime}=e_{2}-\lambda a_{1}+\mu a_{2}, \quad e_{3}^{\prime}=e_{3}
$$

и оставив базис в $\mathfrak{a}$ неизменным, получаем, что центральное расширение эквивалентно самой $T_{2}$.

Расширение $T_{1}$. В общем виде центральное расширение $T_{1}$ записывается следующим образом:

$$
\left[e_{2}, e_{3}\right]=e_{1}+\lambda a_{1}, \quad\left[e_{1}, e_{2}\right]=\mu a_{2}, \quad\left[e_{1}, e_{3}\right]=\nu a_{3},
$$

где $a_{i}$ порождают $\mathfrak{a}$. Возможны три случая.

Первый случай: $\mu, \nu \neq 0$. Выполнив замену

$$
e_{1}^{\prime}=e_{1}+\lambda a_{1}, \quad e_{2}^{\prime}=e_{2}, \quad e_{3}^{\prime}=e_{3}
$$


и оставив базис в $\mathfrak{a}$ неизменным, получаем, что центральное расширение эквивалентно пятимерной алгебре Ли $A_{5,3}$ из списка работы [19], в частности эти алгебры Ли изоморфны.

Второй случай: одна из констант равна нулю. Без ограничения общности считаем, что $\nu=0$. Выполнив замену координат

$$
e_{1}^{\prime}=e_{1}+\lambda a_{1}, \quad e_{2}^{\prime}=e_{2}, \quad e_{3}^{\prime}=e_{3}
$$

и оставив базис в $\mathfrak{a}$, получаем центральное расширение, эквивалентное четырехмерной алгебре Ли $A_{4,1}$ из списка работы [19], в частности эти алгебры Ли изоморфны.

Третий случай: обе константы равны нулю. Легко видеть, что такое центральное расширение эквивалентно $T_{1}$.

Расширение $T_{0}$. В общем виде центральное расширение $T_{0}$ записывается следующим образом:

$$
\left[e_{2}, e_{3}\right]=\lambda a_{1}, \quad\left[e_{1}, e_{2}\right]=\mu a_{2}, \quad\left[e_{1}, e_{3}\right]=\nu a_{3},
$$

где $a_{i}$ порождают $\mathfrak{a}$.

Рассмотрим три случая.

Первый случай: $\lambda, \mu, \nu \neq 0$. После замены координат

$$
a_{1}^{\prime}=\lambda a_{1}, \quad a_{2}^{\prime}=\mu a_{2}, \quad a_{3}^{\prime}=\nu a_{3}
$$

структурные константы полученной алгебры Ли совпадают со структурными константами $A_{6,3}$ из списка работы [19].

Второй случай: одна из констант равна нулю. Без ограничения общности считаем, что $\nu=0$. Выполнив замену координат

$$
a_{1}^{\prime}=\lambda a_{1}, \quad a_{2}^{\prime}=\mu a_{2},
$$

получаем с точностью до переименования базисных векторов алгебру Ли $A_{5,1}$ из списка работы [19].

Третий случай: две из трех констант равны нулю. Без ограничения общности считаем, что $\mu=\nu=0$. Выполнив замену $a_{1}^{\prime}=\lambda a_{1}$, видим, что эта алгебра Ли эквивалентна трехмерной алгебре Гейзенберга $\mathfrak{h}_{3}$, она же $T_{1}$.

Теорема 4 доказана.

\section{§ 6. Задача классификации алгебр Ли с орбитами общего положения размерности 2}

Прежде чем перейти непосредственно к классификации, заметим, что если вместо алгебры Ли g рассматривать прямую сумму этой алгебры с некоторой коммутативной, т.е. центром, то размерность орбит коприсоединенного представления общего положения остается той же самой. Следующая теорема представляет собой основной результат работы. 
ТеОрема 5. С точностъю до прямой суммы с иентром произвольной размерности существуют одна бесконечная серия и шесть исключительных алгебр Ли над полем действительных чисел, размерность орбит коприсоединенного представления общего положения которых равна 2:

1) бесконечная серия - полупрямые суммы вида $\mathbb{R}+{ }_{\rho} \mathbb{R}^{n}$;

2) трехмерная простая алгебра Ли so(3);

3) трехмерная простая алгебра Ли $\mathrm{sl}(2)$;

4) четырехмерная разрешимая алгебра Ли $A_{4,8}$; в подходящем базисе $e_{i}$ она задается коммутационными соотношениями (указаны только ненулевые)

$$
\left[e_{2}, e_{3}\right]=e_{1}, \quad\left[e_{2}, e_{4}\right]=e_{2}, \quad\left[e_{3}, e_{4}\right]=-e_{3} ;
$$

5) четырехмерная разрешимая алгебра Ли $A_{4,10}$; в подходящем базисе $e_{i}$ она задается коммутационными соотношениями (указаны только ненулевые)

$$
\left[e_{2}, e_{3}\right]=e_{1}, \quad\left[e_{2}, e_{4}\right]=-e_{3}, \quad\left[e_{3}, e_{4}\right]=e_{2} ;
$$

6) пятимерная разрешимая алгебра Ли $A_{5,3}$; в подходящем базисе е $e_{i}$ она задается коммутационными соотношениями (указаны только ненулевые)

$$
\left[e_{3}, e_{4}\right]=e_{5}, \quad\left[e_{3}, e_{5}\right]=e_{1}, \quad\left[e_{4}, e_{5}\right]=e_{3}
$$

7) шестимерная нильпотентная алгебра Ли $A_{6,3}$; в подходящем базисе $e_{i}$ она задается коммутационными соотношениями (указаны только ненулевые)

$$
\left[e_{1}, e_{2}\right]=e_{6}, \quad\left[e_{1}, e_{3}\right]=e_{4}, \quad\left[e_{2}, e_{3}\right]=e_{5} .
$$

ДокАЗАТЕЛЬСтво. Пусть размерность орбиты коприсоединенного представления в общем положении алгебры Ли g равна 2. Обозначим размерность самой алгебры через $n+1$. Разберем несколько случаев.

Случай $n=1$. Существует ровно одна некоммутативная алгебра Ли - это $\mathfrak{b}_{2}$. $\mathrm{B}$ подходящем базисе $e_{1}, e_{2}$ единственное коммутационное соотношение в этой алгебре имеет вид $\left[e_{1}, e_{2}\right]=e_{2}$. Понятно, что размерность орбиты коприсоединенного представления общего положения равна 2, а саму алгебру Ли можно включить в серию 1) в условии теоремы: она представляется в виде $\mathbb{R}+{ }_{\rho} \mathbb{R}$.

Случай $n=2$. Полный список трехмерных алгебр Ли приведен в табл. 1. За исключением коммутативной алгебры Ли $T_{0}$ все эти алгебры Ли имеют индекс 1 , т.е. размерность орбиты коприсоединенного представления общего положения равна 2. Из этих алгебр Ли мы также исключим $\mathfrak{b}_{2} \oplus \mathbb{R}$ как уже рассмотренную, а также $\mathrm{sl}(2)$ и so(3) как простые. Покажем, что все оставшиеся алгебры Ли - полупрямые суммы $\mathbb{R}+{ }_{\rho} \mathbb{R}^{2}$. В подходящем базисе $e_{1}, e_{2}, e_{3}$ в $\mathfrak{g}$ и двойственном базисе в $\mathfrak{g}^{*}$ остальные тензоры Пуассона, которые мы объединили в три класса, имеют следующий вид.

Два из них не зависят от параметров:

$$
T_{1}=\left(\begin{array}{ccc}
0 & 0 & 0 \\
0 & 0 & e_{1} \\
0 & -e_{1} & 0
\end{array}\right), \quad T_{2}=\left(\begin{array}{ccc}
0 & 0 & e_{1} \\
0 & 0 & e_{1}+e_{2} \\
-e_{1} & -e_{1}-e_{2} & 0
\end{array}\right) .
$$


$\mathrm{B}$ первом случае $\mathbb{R}$ натянуто на $e_{2}$, a $\mathbb{R}^{2}-$ на $e_{1}, e_{3}$. Представление $\rho$ в этом случае имеет вид

$$
\rho\left(e_{2}\right)=\left(\begin{array}{ll}
0 & 1 \\
0 & 0
\end{array}\right) .
$$

Во втором случае $\mathbb{R}$ натянуто на $e_{3}$, a $\mathbb{R}^{2}-$ на $e_{1}, e_{2}$. Представление $\rho$ в этом случае имеет вид

$$
\rho\left(e_{3}\right)=\left(\begin{array}{ll}
1 & 1 \\
0 & 1
\end{array}\right) .
$$

Еще два тензора зависят от некоторого непрерывного параметра

$$
\begin{gathered}
T_{3, a}=\left(\begin{array}{ccc}
0 & 0 & e_{1} \\
0 & 0 & a e_{2} \\
-e_{1} & -a e_{2} & 0
\end{array}\right), \quad \text { где } 0<|a| \leqslant 1, \\
T_{4, a}=\left(\begin{array}{ccc}
0 & 0 & a e_{1}-e_{2} \\
0 & 0 & e_{1}+a e_{2} \\
-a e_{1}+e_{2} & -e_{1}-a e_{2} & 0
\end{array}\right), \quad \text { где } a \geqslant 0 .
\end{gathered}
$$

Для $T_{3, a}$ подалгебра $\mathbb{R}$ натянута на $e_{3}$, a $\mathbb{R}^{2}-$ на $e_{1}, e_{2}$. Представление $\rho$ в этом случае имеет вид

$$
\rho\left(e_{3}\right)=\left(\begin{array}{ll}
1 & 0 \\
0 & a
\end{array}\right) .
$$

Для $T_{4, a}$ подалгебра $\mathbb{R}$ натянута на $e_{3}$, a $\mathbb{R}^{2}-$ на $e_{1}, e_{2}$. Представление $\rho$ в этом случае имеет вид

$$
\rho\left(e_{3}\right)=\left(\begin{array}{cc}
a & -1 \\
1 & a
\end{array}\right) .
$$

Случай $n>2$. Зафиксируем в алгебре Ли такой базис $e_{1}, \ldots, e_{n+1}$, что $e_{i}, i \geqslant 3$, задает базис в централизаторе некоторого регулярного элемента $a$ Двойственного к $\mathfrak{g}$ пространства, т.е. множество $x \in \mathfrak{g}$ таких, что $\operatorname{ad}_{x}^{*} a=0$. Для $a$ общего положения централизатор коммутативен. Обозначим

$$
l_{i}(x)=\left[e_{1}, e_{i}\right], \quad p_{i}(x)=\left[e_{2}, e_{i}\right], \quad i \geqslant 3, \quad\left[e_{1}, e_{2}\right]=f(x),
$$

где $x$ - координаты точки на $\mathfrak{g}^{*}$ в базисе, двойственном к $e_{1}, \ldots, e_{n+1}$. В этих обозначениях тензор Пуассона имеет следующий вид:

$$
\left(\begin{array}{cccccc}
0 & -f & -l_{3} & -l_{4} & \ldots & l_{n+1} \\
f & 0 & -p_{3} & -p_{4} & \ldots & p_{n+1} \\
l_{3} & p_{3} & 0 & 0 & \ldots & 0 \\
l_{4} & p_{4} & 0 & 0 & \ldots & 0 \\
\vdots & \vdots & \vdots & \vdots & \ddots & 0 \\
l_{n+1} & p_{n+1} & 0 & 0 & \ldots & 0
\end{array}\right) .
$$

Определим на $\mathbb{R}^{n+1}$ пару векторных полей $v$ и $w$ :

$$
v=\left(0,0, l_{3}, \ldots, l_{n+1}\right), \quad w=\left(0,0, p_{3}, \ldots, p_{n+1}\right) .
$$


Если оба векторных поля нулевые, то единственной ненулевой компонентой (алгебра Ли g не может быть коммутативной) в тензоре Пуассона (6.1) является линейный функционал $f=a e_{1}+b e_{2}+f_{0}$, где $a, b$ - константы, а $f_{0}$ - линейный функционал, зависящий от $e_{3}, \ldots, e_{n+1}$. Тут возможны два случая.

Первый случай: $f_{0}=0$. Алгебра Ли $\mathfrak{g}$ представляет собой прямую сумму центра и $\mathfrak{b}_{2}$.

Второй случай: $f_{0} \neq 0$. После подходящей замены координат можно считать, что $f=e_{3}$. Таким образом, мы имеем прямую сумму центра и алгебры Гейзенберга.

Для завершения доказательства теоремы 5 нам потребуются три леммы.

Лемма 3. Векторные поля $v$ и ш зависимы всюоу на $\mathbb{R}^{n+1}$.

ДокАЗАТЕЛЬство. Известно, что почти всюду на $\mathbb{R}^{n+1}$ ранг тензора Пуассона (6.1) равен 2. Из этого вытекает, в частности, что всякий минор размера $4 \times 4$ равен нулю. Рассмотрим минор вида $M_{12 i j}$, где $3 \leqslant i<j$. Он имеет вид

$$
0=M_{12 i j}=\left|\begin{array}{cccc}
0 & -f & -l_{i} & -l_{j} \\
f & 0 & -p_{i} & -p_{j} \\
l_{i} & p_{i} & 0 & 0 \\
l_{j} & p_{j} & 0 & 0
\end{array}\right|= \pm\left|\begin{array}{cc}
l_{i} & p_{i} \\
l_{j} & p_{j}
\end{array}\right|^{2} .
$$

В силу произвольности выбора $i$ и $j$ получаем, что все координаты бивектора $v \wedge w$ равны нулю, т.е. векторные поля всюду зависимы.

Линейный функционал $f_{0}$ может быть нулевым или ненулевым.

Первый случай: векторные поля $v$ и $w$ пропорциональны, т.е. без ограничения общности $v=\lambda w$, где $\lambda$ - некоторая константа (возможно, нулевая). Выполним замену $e_{2}^{\prime}=e_{2}-\lambda e_{1}$, а все остальные векторы базиса оставим без изменений. Легко видеть, что в этом случае $\left[e_{i}^{\prime}, e_{j}^{\prime}\right]=0$ для всех $i, j \geqslant 2$. По теореме 1 получаем, что $\mathfrak{g}$ представляет собой полупрямую сумму $\mathbb{R}$ и $\mathbb{R}^{n}$.

Второй случай: $v=a l(x), w=a p(x)$, где $a$ - некоторый постоянный вектор, a $l(x), p(x)$ - линейные функционалы. Обозначим координаты вектора $a$ через $0,0, a_{3}, \ldots, a_{n+1}$. Без ограничения общности считаем, что $a_{3} \neq 0$ (в противном случае получаем уже разобранный случай - прямую сумму $\mathfrak{b}_{2} \mathrm{c}(n-1)$-мерным центром). Рассмотрим замену координат

$$
e_{i}^{\prime}=e_{i}-\frac{a_{i}}{a_{3}} e_{3}, \quad i \geqslant 4, \quad e_{1}^{\prime}=e_{1}, \quad e_{2}^{\prime}=e_{2}, \quad e_{3}^{\prime}=e_{3} .
$$

Легко видеть, что в новом базисе $\left[e_{i}^{\prime}, e_{j}^{\prime}\right]=0$, где $i \geqslant 4, j=1,2,3$. Таким образом, в этом случае $\mathfrak{g}$ изоморфно центральному расширению некоторой трехмерной алгебры Ли. Среди центральных расширений трехмерных алгебр Ли мы еще не рассмотрели только $A_{4,10}, A_{4,8}, A_{4,1}, A_{5,1}, A_{5,3}, A_{6,3}$.

ЛЕмма 4. Алгебры Ли $A_{4,1}$ и $A_{5,1}$ являются полупрямыми суммами $\mathbb{R}$ с пространствами $\mathbb{R}^{3}$ и $\mathbb{R}^{4}$ соответственно.

ДокАзАтЕльство. Ненулевые коммутационные соотношения для $A_{4,1}$ в доказательстве теоремы 4 имеют вид

$$
\left[e_{2}, e_{3}\right]=e_{1}, \quad\left[e_{1}, e_{3}\right]=a_{3} .
$$


$\mathrm{B}$ этом базисе одномерное пространство $\mathbb{R}$, натянутое на $e_{3}$, действует на трехмерном пространстве $\mathbb{R}^{3}$, натянутом на векторы $e_{2}, e_{1}, a_{3}$. Оператор $\operatorname{ad}_{e_{3}}$ имеет вид

$$
\left(\begin{array}{lll}
0 & 1 & 0 \\
0 & 0 & 1 \\
0 & 0 & 0
\end{array}\right)
$$

Ненулевые коммутационные соотношения для $A_{5,1}$ в доказательстве теоремы 4 имеют вид

$$
\left[e_{3}, e_{2}\right]=a_{1}, \quad\left[e_{1}, e_{2}\right]=a_{2} .
$$

$\mathrm{B}$ этом базисе одномерное пространство $\mathbb{R}$, натянутое на $e_{2}$, действует на четырехмерном пространстве $\mathbb{R}^{4}$, натянутом на векторы $e_{1}, a_{2}, e_{3}, a_{1}$. Оператор $\operatorname{ad}_{e_{3}}$ имеет вид

$$
\left(\begin{array}{llll}
0 & 1 & 0 & 0 \\
0 & 0 & 0 & 0 \\
0 & 0 & 0 & 1 \\
0 & 0 & 0 & 0
\end{array}\right)
$$

Лемма доказана.

Лемма 5. Ни одна из четырех попарно не изоморфных алгебр Ли $A_{4,10}$, $A_{4,8}, A_{5,3}, A_{6,3}$ не является полупрямой суммой вида $\mathbb{R}+{ }_{\rho} \mathbb{R}^{n}$.

ДокАзАтЕльство. Для начала заметим, что коммутатор алгебры Ли $\mathbb{R}+{ }_{\rho} \mathbb{R}^{n}$ лежит в коммутативной подалгебре $\mathbb{R}^{n}$ и, как следствие, коммутативен. В случаях $A_{4,8}$ и $A_{4,10}$ коммутатор изоморфен некоммутативной алгебре Гейзенберга $\mathfrak{h}_{3}$, поэтому они не являются полупрямыми суммами одномерной и трехмерной коммутативных алгебр Ли.

Рассмотрим алгебру Ли $A_{5,3}$. Ненулевые коммутационные соотношения для нее в доказательстве теоремы 4 имеют вид

$$
\left[e_{2}, e_{3}\right]=e_{1}, \quad\left[e_{1}, e_{2}\right]=a_{2}, \quad\left[e_{1}, e_{3}\right]=a_{3} .
$$

Предположим, что эта подалгебра представляется в виде полупрямой суммы $\mathbb{R}+{ }_{\rho} \mathbb{R}^{4}$. Заметим, что коммутатор $A_{5,3}$ имеет размерность 3 , поэтому эта подалгебра заведомо не может представляться в виде $\mathfrak{h}_{3} \oplus \mathbb{R}^{n-2}$ или $\mathfrak{b}_{2} \oplus \mathbb{R}^{n-1}$. Из этого по теореме 1 получаем, что в данной алгебре коммутативная подалгебра размерности 4, которую мы обозначим $\mathfrak{a}$, представляет собой идеал и единственна. При этом она, разумеется, содержит центр $\mathfrak{z}$, натянутый на $a_{2}, a_{3}$.

Перейдем к факторалгебре $\mathfrak{g} / \mathfrak{z}$, которая изоморфна $\mathfrak{h}_{3}$. При этом $\mathfrak{a} / \mathfrak{z}$ представляет собой коммутативный идеал размерности 2 в $\mathfrak{h}_{3}$. Это означает, что он натянут на $e_{1}$ и произвольный вектор вида $\lambda e_{2}+\mu e_{3}$. Из этого следует, что $\mathfrak{a}$ натянут на $a_{2}, a_{3}, e_{1}, \lambda e_{2}+\mu e_{3}$. Из двух последних коммутационных соотношений для $A_{5,3}$ следует, что этот идеал коммутативен тогда и только тогда, когда $\lambda=\mu=0$. Следовательно, идеал имеет размерность 3 - противоречие с утверждением теоремы 1 . Стало быть, $A_{5,3}$ не является полупрямой суммой одномерной и четырехмерной коммутативных алгебр Ли. 
Теперь рассмотрим $A_{6,3}$. Ее коммутационные соотношения имеют вид

$$
\left[e_{2}, e_{3}\right]=a_{1}, \quad\left[e_{1}, e_{2}\right]=a_{2}, \quad\left[e_{1}, e_{3}\right]=a_{3} .
$$

Пусть $A_{6,3}$ представляется в виде полупрямой суммы $\mathbb{R}+\rho \mathbb{R}^{5}$. Так как алгебpa $A_{6,3}$ нильпотентна, то оператор $\rho\left(e_{1}\right)$ также нильпотентен. Так как коммутатор имеет размерность 3 , то такова же размерность образа оператора $\rho\left(e_{1}\right): \mathbb{R}^{5} \rightarrow \mathbb{R}^{5}$. Как следствие, в нормальной жордановой форме оператора - ровно две жордановы клетки. При этом размер одной должен быть, как минимум, 3. Так как факторалгебра по центру коммутативна, то легко видеть, что в нормальной жордановой форме оператора $\rho\left(e_{1}\right)$ не может быть клеток размерности больше 2. Противоречие. Лемма доказана.

Доказательство леммы 5 завершает доказательство теоремы 5 .

\section{Список литературы}

[1] А. В. Болсинов, А. М. Изосимов, А. Ю. Коняев, А. А. Ошемков, “Алгебра и топология интегрируемых систем. Задачи для исследования”, Труды семинара по векторному и тензорному анализу с их приложениями к геометрии, механике и физике, 28, ред. А. А. Ошемков, Изд-во Моск. ун-та, М., 2012, 119-191.

[2] A. V. Bolsinov, I. A. Taimanov, "Integrable geodesic flows with positive topological entropy", Invent. Math., 140:3 (2000), 639-650.

[3] А. В. Болсинов, И. А. Тайманов, "Интегрируемые геодезические потоки на надстройках автоморфизмов торов", Динамические системы, автоматы и бесконечные группы, Сборник статей, Тр. МИАН, 231, Наука, М., 2000, 46-63; англ. пер.: A. V. Bolsinov, I. A. Taimanov, "Integrable geodesic flows on suspensions of automorphisms of tori", Proc. Steklov Inst. Math., 231 (2000), 42-58.

[4] A. S. Mishchenko, A. T. Fomenko, "Symplectic Lie group actions", Algebraic topology, Aarhus 1978, Proc. Sympos. (Univ. Aarhus, Aarhus, 1978), Lecture Notes in Math., 763, Springer-Verlag, 1979, 504-538.

[5] А. Т. Фоменко, "О симплектических структурах и интегрируемых системах на симметрических пространствах", Матем. сб., 115(157):2(6) (1981), 263-280; англ. пер.: А. Т. Fomenko, "On symplectic structures and integrable systems on symmetric spaces", Math. USSR-Sb., 43:2 (1982), 235-250.

[6] В. В. Трофимов, А. Т. Фоменко, "Интегрируемость по Лиувиллю гамильтоновых систем на алгебрах Ли", УМH, 39:2(236) (1984), 3-56; англ. пер.: V. V. Trofimov, A.T. Fomenko, "Liouville integrability of Hamiltonian systems on Lie algebras", Russian Math. Surveys, 39:2 (1984), 1-67.

[7] А. В. Браилов, А. Т. Фоменко, “Топология интегральных подмногообразий вполне интегрируемых гамильтоновых систем", Матем. сб., 134(176):3(11) (1987), 375-385; англ. пер.: A.V. Brailov, A. T. Fomenko, "The topology of integral submanifolds of completely integrable Hamiltonian systems", Math. USSR-Sb., 62:2 (1989), 373-383.

[8] A. T. Fomenko, V.V. Trofimov, Integrable systems on Lie algebras and symmetric spaces, Adv. Stud. Contemp. Math., 2, Gordon and Breach Science Publishers, New York, 1988, xii+294 pp.

[9] А. Т. Фоменко, "Симплектическая топология вполне интегрируемых гамильтоновых систем", УМH, 44:1(265) (1989), 145-173; англ. пер.: А. Т. Fomenko, "The symplectic topology of completely integrable Hamiltonian systems", Russian Math. Surveys, 44:1 (1989), 181-219. 
[10] В. В. Трофимов, А. Т. Фоменко, Алгебра и геометрия интегрируемых гамильтоновых дифференциальных уравнений, Математика и ее приложения, Факториал, М.; Изд-во Удмуртского гос. ун-та, Ижевск, 1995, 448 с.

[11] D. Panyushev, O. Yakimova, "The argument shift method and maximal commutative subalgebras of Poisson algebras", Math. Res. Lett., 15:2 (2008), 239-249.

[12] А.В. Болсинов, "Согласованные скобки Пуассона на алгебрах Ли и полнота семейств функций в инволюции", Изв. АН СССР. Сер. матем., 55:1 (1991), 68-92; англ. пер.: A. V. Bolsinov, "Compatible Poisson brackets on Lie algebras and completeness of families of functions in involution", Math. USSR-Izv., 38:1 (1992), 69-90.

[13] C. А. Шашков, "Коммутативные однородные пространства с одномерным стабилизатором", Изв. РАН. Сер. матем., 76:4 (2012), 185-206; англ. пер.: S. A. Shashkov, "Commutative homogeneous spaces with one-dimensional stabilizer", Izv. Math., 76:4 (2012), 820-839.

[14] D. Arnal, M. Cahen, J. Ludwig, "Lie groups whose coadjoint orbits are of dimension smaller or equal to two", Lett. Math. Phys., 33:2 (1995), 183-186.

[15] Г. М. Мубаракзянов, "Некоторые теоремы о разрешимых алгебрах Ли", Изв. вузов. Матем., 1966, №6, 95-98.

[16] А. В. Борисов, А. А. Килин, И. С. Мамаев, "Гамильтоновость и интегрируемость задачи Суслова", Нелинейная динам., 6:1 (2010), 127-142.

[17] J.E. Marsden, T.S. Ratiu, Introduction to mechanics and symmetry. A basic exposition of classical mechanical systems, 2nd ed., Texts Appl. Math., 17, Springer-Verlag, New York, 1999, xviii+582 pp.

[18] L. Bianchi, "Sugli spazi a tre dimensioni che ammettono un gruppo continuo di movimenti (citeulike:3081031)", Mem. Mat. Fis. Soc. Ital. Sci. (3), 11 (1898), 267-352.

[19] J. Patera, R. T. Sharp, P. Winternitz, H. Zassenhaus, "Invariants of real low dimension Lie algebras", J. Mathematical Phys., 6:17 (1976), 986-994.

[20] А. А. Короткевич, "Интегрируемые гамильтоновы системы на алгебрах Ли малой размерности", Матем. сб., 200:12 (2009), 3-40; англ. пер.: А. А. Korotkevich, "Integrable Hamiltonian systems on low-dimensional Lie algebras", Sb. Math., 200:12 (2009), 1731-1766.

\section{Андрей Юрьевич Коняев (Andrei Yu. Konyaev)}

Механико-математический факультет

Московского государственного университета

им. М. В. Ломоносова

E-mail: maodzund@bk.ru
Поступила в редакцию 10.11.2012 и 15.10 .2013 